\title{
Regulating dynamic signaling between hematopoietic stem cells and niche cells via a hydrogel matrix
}

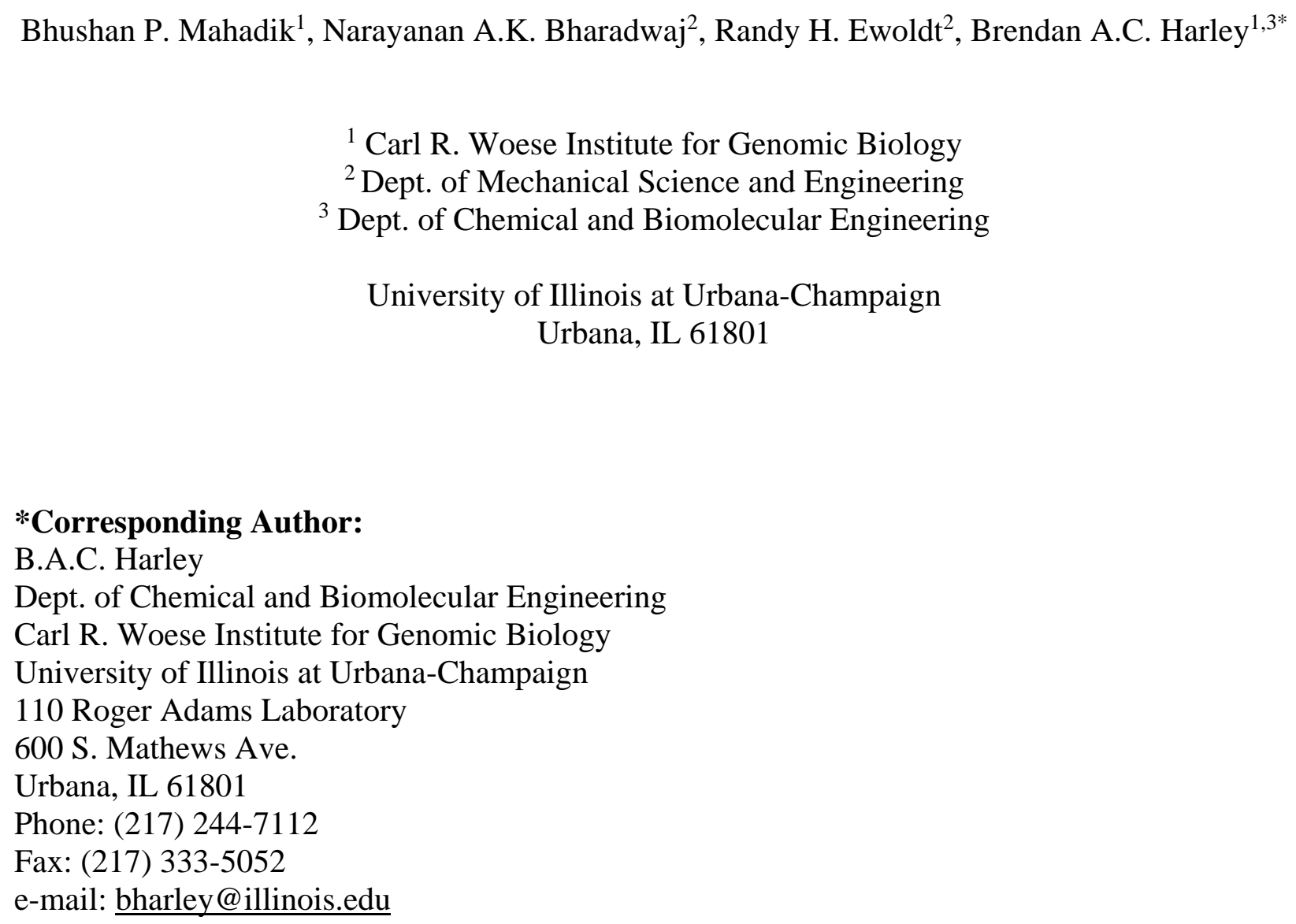




\section{ABSTRACT}

2 Hematopoietic stem cells (HSC) reside in unique bone marrow niches and are influenced by

3 signals from surrounding cells, the extracellular matrix (ECM), ECM-bound or diffusible

4 biomolecules. Here we describe the use of a three-dimensional hydrogel to alter the balance of

5 HSC-generated autocrine feedback and paracrine signals generated by co-cultured niche-

6 associated cells. We report shifts in HSC proliferation rate and fate specification in the presence

7 of lineage positive $\left(\mathrm{Lin}^{+}\right)$niche cells. Hydrogels promoting autocrine feedback enhanced

8 expansion of early hematopoietic progenitors while paracrine signals from $\mathrm{Lin}^{+}$cells increased

9 myeloid differentiation. We report thresholds where autocrine vs. paracrine cues alter HSC fate

10 transitions, and were able to selectively abrogate the effects of matrix diffusivity and niche cell

11 co-culture via the use of inhibitory cocktails of autocrine or paracrine signals. Together, these

12 results suggest diffusive biotransport in three-dimensional biomaterials are a critical design

13 element for the development of a synthetic stem cell niche.

14

15 Keywords: hematopoietic, hydrogel diffusivity, autocrine, paracrine, signaling

16

17

18

19

20

21 


\section{1. Introduction}

2 Hematopoietic stem cells (HSCs) are responsible for mediating the process of hematopoiesis, the

3 continuous generation of the body's full complement of blood and immune cells. These events

4 take place in unique regions of the bone marrow termed niches [1-6]. While the genetic

5 information required to direct HSC fate specification events such as quiescence, self-renewal, or

6 differentiation is contained within its DNA, signals from the niche - surrounding cells, the

7 extracellular matrix (ECM), ECM-bound or diffusible biomolecules - trigger these events [7-9].

8 An artificial bone marrow would have significant clinical value, both for therapeutic expansion

9 of HSCs to improve hematopoietic stem cell transplants and to facilitate studies of the etiology

10 and treatment of hematologic diseases.

12 Recent efforts have focused on the development of biomaterials that regulate the presentation of

13 matrix-based signals (e.g., stiffness, composition) or exogenously-provided (soluble or matrix

14 bound) growth factors [10-13] to direct HSC fate. However, exploring the role played by

15 interactions between HSCs and supportive niche cells within the matrix provides a new avenue

16 for investigation. Direct cell-cell interactions (juxtacrine signals) rely on the activation of

17 membrane-bound cell adhesion molecules; these signals have been exploited in a biomaterial

18 context to alter HSC survival and differentiation $[14,15]$. Indirect cell-cell interactions between

19 HSCs and niche cells (paracrine signals) or autocatalytic effects from the HSC itself (autocrine

20 signals) are mediated by diffusible biomolecules [16-20]. Recently, experimental and modeling

21 efforts have begun to examine niche-inspired paracrine and autocrine signals, primarily using

22 liquid culture systems [19, 21]. While demonstrating the potential to alter HSC lineage 
1 specification profiles, translation of such systems into fully three-dimensional biomaterial

2 platforms offers an opportunity for innovation but remains poorly explored.

4 Within the niche, HSCs and supportive niche cells secrete and subsequently interact with a large 5 number of cytokines and growth regulators [22]. Mesenchymal stem cells (MSCs) are believed

6 to stimulate proliferation of HSCs through paracrine-mediated interactions via several molecules

7 such as CXCL12, IL-6 and TPO [20]. Csaszar et al. demonstrated that paracrine-mediated IL-6

8 signaling between $\mathrm{CD} 34^{+}$human progenitor cells can be regulated by activation of the delta- 1

9 ligand and is responsible for HSC expansion [19]. Similarly, the transforming growth factor $\beta$

10 (TGF- $\beta$ ) pathway has been shown to be activated via both niche generated paracrine signals and

11 HSC-generated autocrine feedback to inhibit HSC self-renewal and proliferation [16, 23]. In

12 vitro autocrine feedback activated via vascular endothelial growth factor (VEGF) have also been

13 shown to maintain HSC survival [17] while platelet derived growth factor (PDGF) mediated

14 autocrine feedback can enhance proliferation [23, 24]. From an engineering context, Zandstra et

15 al. demonstrated selective inhibition of paracrine and autocrine feedback signal transduction

16 pathways in liquid culture to alter HSC fate [21, 23, 25]. Recently, Müller et al. described the use

17 of arrays of microcavities to culture single or small groups of hematopoietic stem and progenitor

18 cells (HSPCs), and reported that while autocrine feedback within the microcavity culture may

19 play a role in HSC quiescence, paracrine signaling provided both stimulatory and inhibitory

20 effects [26]. While these studies highlight the importance of autocrine and paracrine signaling

21 mechanisms for HSC biomanufacturing, significant opportunity exists to develop a framework to

22 examine the balance of these signals within a fully-3D biomaterial platform where matrix

23 diffusive transport plays a critical role. 
2 Here we study the coordinated effects of paracrine signals produced by co-encapsulated $\mathrm{Lin}^{+}$

3 niche cells and HSC-generated autocrine feedback on HSC lineage specification. We

4 hypothesize that the mode of cell communication in a diffusion-restricted environment is a

5 function of niche cell density, responsible for establishing the concentration of paracrine signals,

6 and the diffusivity of the hydrogel encapsulating the cells. We further hypothesize that altering

7 the delivery of paracrine signals from niche cells or the balance of diffusive loss vs. retention of

8 HSC-generated autocrine signals alters HSC fate specification. We explored this concept using

9 the well-described murine hematopoietic stem cell system, choosing primary bone marrow

10 derived $\mathrm{Lin}^{-} \mathrm{Scal}^{+} \mathrm{cKit}^{+}$(LSK) sub-fraction as HSCs and $\mathrm{Lin}^{+}$marrow cells as supportive niche

11 cells known to secrete biomolecular cues that stimulate HSC proliferation and lineage

12 specification [23]. HSCs were encapsulated with $\mathrm{Lin}^{+}$niche cells in collagen hydrogels, varying

13 both hydrogel density $(1,3 \mathrm{mg} / \mathrm{mL})$ and $\operatorname{HSC}: \operatorname{Lin}^{+}$ratio $(1: 0,1: 10,1: 100)$, with liquid culture

14 used as a control (Fig. 1). Cultures were limited to 2 days in order to explicitly consider early

15 HSC responses to mixed cultures and to limit the effects of dynamic feedback responses. We

16 traced HSC response via established metrics of HSC apoptosis, proliferation, surface antigen

17 expression, and colony forming unit capacity, and used selective inhibitors of paracrine and

18 autocrine signaling pathways to validate our findings. We report HSC fate specification as a

19 function of diffusion-restricted (autocrine feedback dominated) vs. diffusion unlimited (paracrine

20 dominated) biomaterial environments, and in so doing define a biomaterial-based approach to

21 regulate the balance of autocrine vs. paracrine signals to tune HSC proliferation vs.

22 differentiation. 


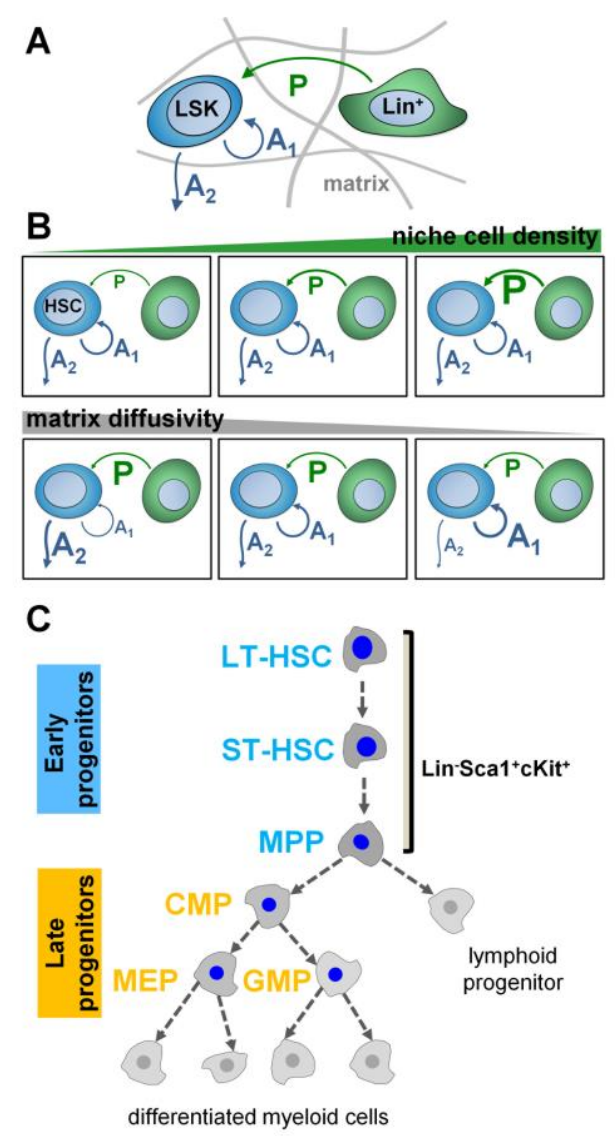

Figure 1. Hematopoietic stem cell - Lin+ niche cell interactions within a hydrogel niche. (A) A schematic depicting biomolecular interactions between HSCs and niche cells encapsulated within a hydrogel matrix. P: paracrine signals generated by the niche cell population. A1: Fraction of HSC-generated autocrine signals that contribute to feedback loop. A2: Fraction of HSC-generated autocrine signals that diffuse away. (B) Schematic of expected effects of changes in niche cell density versus matrix diffusivity on the magnitude of Paracrine vs. Autocrine feedback reaching the HSC population. Magnitude of signal depicted by the arrow width and font size. (C) Representative image of the HSC differentiation hierarchy depicting the starting $\mathrm{Lin}-\mathrm{Sca} 1^{+} \mathrm{CKit}^{+}$(LSK) fraction along with the early vs. late hematopoietic progenitor cell populations. LT-HSC: Long-term repopulating HSC (LSK·CD34-Flk2'). ST-HSC: Short-term repopulating HSC. MPP: Multipotent progenitor (LSK·CD34·Flk2'). CMP: common myeloid progenitor. MEP: megakaryocyte-erythrocyte progenitor. GMP: granulocyte-macrophage progenitor.

\section{Methods and Materials}

\subsection{HSPC and Lin ${ }^{+}$cell isolation}

4 All work involving primary cells was conducted under approached animal welfare protocols

5 (Institutional Animal Care and Use Committee, University of Illinois at Urbana-Champaign).

6 Primary HSPCs were isolated from the bone marrow of the femur and tibia of female C57BL/6 
1 mice (Jackson Labs; Ages $1-3$ months) as described previously[27]. HSPCs were identified as

2 the $\mathrm{Lin}^{-} \mathrm{Sca}-1^{+} \mathrm{c}-\mathrm{kit}^{+}$(LSK) fraction by incubating the remaining bone marrow cells with a

3 cocktail of antibodies (eBioscience San Diego, CA): PE-conjugated Sca-1 (1:100 dilution), APC-

4 Cy7 conjugated c-kit (1:100 dilution), and a 1:100 dilution of a FITC-conjugated Lineage (Lin)

5 cocktail (CD5, B220, Mac-1, CD8a, Gr-1, Ter-119). Both the LSK and Lin ${ }^{+}$fraction was sorted

6 using a BD FACS Aria II flow cytometer (BD FACS Diva software) and collected in PBS/FBS

7 on ice for immediate use.

$9 \quad 2.2$ Collagen hydrogel preparation

10 Rat tail type I collagen (BD Biosciences, Bedford MA) was used to make the cell-laden collagen

11 hydrogels. Collagen solutions of defined densities $(1,3 \mathrm{mg} / \mathrm{mL})$ were prepared from the stock

12 collagen solution of approximately $9 \mathrm{mg} / \mathrm{mL}$ (lot specific). The final concentrations were as

13 follows: 1x M199 buffer (supplemented with 0.75\% NaHCO2 and 0.01 M HEPES) in a ratio of

14 1:4 with collagen stock; $1 \mathrm{mM}$ Dithiothreitol (DTT, Sigma); $5 \mathrm{mM} \mathrm{CaCl2;} 0.1 \mathrm{mM}$ Glyoxal; $0.4 \mathrm{M}$

$15 \mathrm{NaOH}$ added to adjust $\mathrm{pH}$ between 7.3-7.4 [28]. Final gel volume was achieved by adding the

16 cell-media suspension to create a $60 \mu \mathrm{L}$ solution that was pipetted into a 96 well plate. Gelation

17 was achieved within $1 \mathrm{hr}$ of placing the gels in a humidified incubator at $37{ }^{\circ} \mathrm{C}$ and $5 \% \mathrm{CO}_{2}$.

\subsection{Hydrogel biophysical characterization}

20 Bulk rheological properties of the hydrogels were tested using a torque-controlled rotational

21 rheometer (Discovery Series Hybrid Rheometer (DHR), model HR-3, TA Instruments) using a

22 cone and plate geometry ( $60 \mathrm{~mm}$ cone diameter; $0.5^{\circ}$ cone angle). The base plate was

23 maintained at $37^{\circ} \mathrm{C}$ via a Peltier system, while a solvent trap was used to maintain hydrogel 
1 samples in a humid environment. The collagen network was allowed to evolve in the confines of

2 the cone-plate rheometer setup for $3600 \mathrm{~s}$ and small oscillations in shear ( $3 \mathrm{rad} / \mathrm{s}, 1 \%$ strain

3 amplitude) in order to monitor gelation kinetics and to determine final viscoelastic properties

4 (Supplementary Fig. 2). Following gelation, hydrogels were subjected to an oscillation

5 frequency sweep at 1\% strain amplitude (corresponding to the linear viscoelastic regime as

6 determined via previous oscillatory strain sweep; data not shown). Additionally, step stress tests

7 (to measure creep compliance) were used to characterize longer time behavior (up to $100 \mathrm{~s}$ ) to

8 validate that the hydrogels were soft viscoelastic solids.

10 Hydrogel diffusivity was measured via fluorescence recovery after photobleaching (FRAP)

11 experiments using FITC-conjugated Dextran (40 kDa and 2000 kDa, Sigma Aldrich). Collagen

12 solutions ( 1 and $3 \mathrm{mg} / \mathrm{mL}$ ) were prepared as described above and incubated for $1 \mathrm{hr}$. The gels

13 were incubated with FITC-conjugated Dextran solution overnight. Prior to analysis, the gels

14 were gently washed with PBS. FRAP measurements were performed using the Zeiss 710

15 Multiphoton confocal microscope as described previously [29]. Briefly, a $50 \mu \mathrm{m}$ diameter spot

16 was bleached (Laser intensity: $0.05 \mathrm{~mW}$ ) at the center of the gel. The sample was bleached for 5

17 seconds and recovery due to diffusive transport of fluorescent molecules from the surrounding

18 hydrogel was tracked for up to 15 minutes via fluorescent imaging. The half time $\left(t_{1 / 2}\right)$ of the

19 recovery was calculated using the FRAP function available in the Zeiss software. The hydrogel

20 diffusion coefficient (D) was subsequently calculated as:

$21 \quad D=\frac{w^{2}}{4 t_{1 / 2}}$ 
1 where $D$ is the diffusion coefficient, $w$ the photobleaching beam radius, and $t_{1 / 2}$ the recovery half

2 time [30]. A maximum of 3 spots were bleached per sample, spaced away from each other to

3 minimize effects of the previous photobleaching.

\subsection{Mesh size estimation}

6 To estimate the ratio of the average network mesh size $\xi$ for varying collagen concentrations

7 with fiber diameter $d$, we use the relation $G_{0} \sim \xi^{-22 / 5} d^{28 / 5}$ from an existing theory for semi-

8 flexible polymer networks by Mackintosh et al. [31], and scaling relations for structural elements

9 of collagen proposed by Yang et al. [32]. Using the measured value of $G_{0} \approx 5 \mathrm{~Pa}$ for $1 \mathrm{mg} / \mathrm{mL}$

10 collagen, and $G_{0} \approx 56 \mathrm{~Pa}$ for $3 \mathrm{mg} / \mathrm{mL}$ collagen, and assuming no significant change in the fiber

11 diameter with change in collagen concentration, we estimate $\xi_{3 \mathrm{mg} / \mathrm{mL}} \sim 0.58 \cdot \xi_{1 \mathrm{mg} / \mathrm{mL}}$. The accuracy

12 of these estimates can be verified with mesh size estimates reported by Arevalo et al. via

13 confocal reflectance imaging, predicting $\xi_{3 \mathrm{mg} / \mathrm{mL}} \sim 0.7 \xi_{1 \mathrm{mg} / \mathrm{mL}}$ [33]. This is close to our estimate

14 using the network elastic moduli for these concentrations. It is worth noting that the pore size

15 estimates from theories using diffusion in collagen gels are more accurate and representative of

16 the true mesh size, and are much smaller than that reported using CRM imaging. These studies

17 however do not report mesh sizes for $1 \mathrm{mg} / \mathrm{mL}$ collagen. Determining the actual mesh sizes

18 require additional structural information such as fiber diameter, which is unavailable. Yet, this

$1940 \%$ decrease is consistent with existing reports from confocal reflectance imaging for the same

20 range of concentrations [33].

\section{$22 \quad 2.5$ Dextran hydrodynamic radius estimation}


1 The Mark-Houwink equation relates the average molecular weight of the polymer $M_{w}$ to its

2 intrinsic viscosity $[\eta]$ :

$3[\eta]=K M_{w}^{\alpha}$,

4 where $K$ and $\alpha$ are polymer constants and have been determined for dextran, $K=49.3 \times 10^{-3} \mathrm{~mL} / \mathrm{g}$

5 and $\alpha=0.6$ [34]. For a polymer in solution, the radius of gyration $R_{g}$ is given by [35]:

$$
R_{g}=\left(\frac{[\eta] M_{\mathrm{w}}}{6.2 N_{a}}\right)^{\frac{1}{3}}
$$

7 where $N_{\mathrm{a}}$ is the Avagadro number. The diffusion coefficient $D$ is related to the polymer

8 hydrodynamic radius $R_{\mathrm{h}}$ and the radius of gyration $R_{g}$ as [36]:

$$
D=\frac{k T}{6 \pi \eta_{s} R_{h}}=\frac{0.203 k T}{\sqrt{6} \eta_{s} R_{g}},
$$

10 where $\eta_{\mathrm{s}}$ is the solvent viscosity. For the $40 \mathrm{kDa}$ Dextran $\left(\mathrm{M}_{\mathrm{w}}=40000 \mathrm{~g} / \mathrm{mol}\right)$, we find $D_{37^{\circ} \mathrm{C}}=$

$115.37 \times 10^{-7} \mathrm{~cm}^{2} / \mathrm{s}$ and $R_{\mathrm{h}}=4.2 \mathrm{~nm}$, and for the $2000 \mathrm{kDa}$ Dextran $\left(\mathrm{M}_{\mathrm{w}}=2,000,000 \mathrm{~g} / \mathrm{mol}\right)$, we find

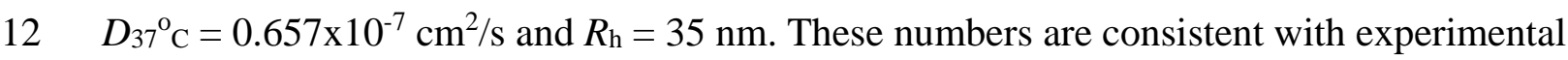

13 reports from other studies [37].

\subsection{Determination of characteristic diffusion time $\left(t_{D}\right)$}

16 The cell-cell diffusion time for cell-secreted biomolecules for each co-culture condition was

17 estimated as follows. For 3D culture, a homogeneous distribution of cells within the $60 \mu \mathrm{L}$

18 hydrogel and a simple cubic lattice structure arrangement of cells within the culture volume was

19 assume. For a cell radius R, surface-surface cell spacing a, total number of cells $\mathrm{N}$, and total

20 hydrogel volume of $60 \mu \mathrm{L}$ :

$$
N *(2 R+a)^{3}=60 \mu L
$$


1 Therefore, we can solve for the cell spacing for each HSC:niche cell condition (1:0, 1:10, and

2 1:100). Further, biomolecular diffusion time ( $t_{D}$; Supplementary Table 1) for a given hydrogel

3 diffusivity $(D)$ is given by:

4

5

6

7 LSKs were seeded in monoculture and co-culture with $\mathrm{Lin}^{+}$bone marrow cells in 2D or 3D

\subsection{Experimental culture conditions} culture environments under the following conditions: LSK:Lin ${ }^{+}$cells at 1:0, 1:10, 1:100, 1:500 in 2D (liquid), $1 \mathrm{mg} / \mathrm{mL}, 3 \mathrm{mg} / \mathrm{mL}$ collagen gels. 5000 LSK cells were used for each condition and cultured for 2 days prior to analysis. The cells were cultured in StemSpan SFEM media (Stemcell Technologies Inc., Vancouver, Canada) supplemented with $100 \mathrm{ng} / \mathrm{mL}$ Stem cell factor (SCF) (PeproTech, Rocky Hill, NJ).

2.8 Surface antigen expression analysis to determine cell phenotype

Cell phenotype was determined via surface antigen expression using flow cytometry on a BD

LSRFortessa flow cytometer (BD Bioscience). Prior to analysis, the collagen gels were digested

using a $50 \mathrm{U} / \mathrm{mL}$ solution of type IV collagenase (Worthington Biochemical) for roughly 30

mins in the incubator. The cells were then washed, filtered and resuspended in $100 \mu \mathrm{L}$ PBS with 5\% FBS (PBS/FBS) and $0.1 \%$ sodium azide. The cell suspension was then stained with a cocktail of the following antibodies (eBioscience): APC anti-CD34 (1:20 dilution, 60 mins), AmCyan anti-IL7R $\alpha$ (1:20, 60 mins BD Bioscience), APC-Cy7 anti-c-kit (1:100, 30 mins), PE anti-Sca-1 (1:100, 30 mins), PE-Cy7 anti-CD16/32 (1:100, 30 mins), PE-Cy5 anti-Flk2 (1:20, 30 mins), FITC-anti lineage cocktail (CD5, B220, Mac-1, CD8a, Gr-1, Ter-119, 1:100, 30 mins).

$$
t_{D}(s)=\frac{a^{2}\left(\mu m^{2}\right)}{D\left(\mu m^{2} / s\right)}
$$


1 The surface antigen-based identification of the cells for all the progenitor sub-fractions is given

2 in Supplementary Table 2.

$4 \quad 2.9$ LSK progenitor identification

5 The LSK progenitors were identified post culture by staining the initial LSK population with the

6 CellTrace Violet Cell Proliferation dye (Invitrogen, Carlsbad, CA) as per manufacturer

7 instructions. Briefly, cells were washed and resuspended in PBS. CellTrace Violet tracking dye

8 was added at a final dye concentration of $1 \mu \mathrm{M}$ for $20 \mathrm{mins}$ at $37^{\circ} \mathrm{C}$. Cells were quenched with

9 cold PBS/FBS, incubated on ice for 5 mins followed by a wash with PBS. LSK progenitors were

10 identified along with other surface markers on the flow cytometer. Progenitor sub-fractions were

11 then calculated as a fraction of these LSK progenitors.

\section{$13 \quad 2.10$ Apoptosis assay}

14 Cells undergoing apoptosis were detected via staining with APC-labeled Annexin V as per

15 manufacturer protocols [38]. Briefly, cells were washed and resuspended in $100 \mu \mathrm{L}$ of $1 \mathrm{X}$

16 Binding Buffer with $1 \%$ FBS and $5 \mu \mathrm{L}$ of the dye. The cells were stored in the dark at room

17 temperature for 30 mins and analyzed within $1 \mathrm{hr}$. As a positive control, cells were treated with 1

$18 \mu \mathrm{M}$ of Staurosporine. To further distinguish between live and dead cells, co-staining with

19 propidium iodide (PI) was performed. A high percentage of cells that stained positive for

20 Annexin V also stained positive for PI. To avoid spectral interference of PI with the surface

21 antigen dyes PE-Cy7 and Cy5, only the Annexin V dye was added to distinguish between live

22 and apoptotic cells. All cell samples were analyzed on a BD LSRFortessa flow cytometer (BD

23 Bioscience) and using the FCS Express 4.0 software. 


\section{$2 \quad 2.11$ Colony-forming unit (CFU) assay}

3 Colony forming potential was assessed via Methocult GF M3434 methylcellulose-based medium

4 (StemCell Technologies, Vancouver, Canada). For this purpose, cells were isolated from

5 collagen hydrogels after culture via collagen digestion mentioned above, filtered, and then

6 resuspended in StemSpan SFEM media. Aliquots were made at one-third and one-fifth of the

7 total sample volume, brought to a volume of $0.1 \mathrm{~mL}$ and mixed with the Methocult media for a

8 total volume of $1.1 \mathrm{~mL}$ per sample. The samples were plated on a gridded $35 \mathrm{~mm}$ culture dishes

9 (StemCell Technologies) and incubated for 12 days. Following incubation, the GEMM, GM, G,

$10 \mathrm{M}, \mathrm{Mk}$, and $\mathrm{E}$ colonies were counted using an inverted microscope. The results are reported as

11 the number of colonies counted per 1000 of the original LSK cells seeded for each condition.

\section{$13 \quad 2.12$ Inhibitor molecules}

14 Small molecule inhibitors for paracrine signaling were added to the $1 \mathrm{mg} / \mathrm{mL}$ collagen condition

15 while inhibitors for autocrine signaling were added to the $3 \mathrm{mg} / \mathrm{mL}$ collagen condition as listed

16 in Supplementary Table 3.

$18 \quad 2.13$ Statistical analysis

19 Statistical analysis was performed via one-way analysis of variance (ANOVA) tests after which

20 a Tukey-HSD post-hoc test was used. Independent factors included niche cell density and

21 hydrogel density. Analysis of LSK cell bioactivity (viability, proliferation index, surface antigen

22 expression, CFU assay) used $n=5$ constructs per group. Significance was set at $p<0.05$. Error

23 bars are reported as standard error of the mean unless otherwise noted. 
2 3. Results

$3 \quad 3.1$ The presence of $\mathrm{Lin}^{+}$niche cells alters HSC progenitor fractions in liquid culture

4 Liquid culture was first used to determine the magnitude of the effect of $\mathrm{Lin}^{+}$niche cell co-

5 culture on HSC response in a diffusion-unrestricted environment. Here, $\mathrm{Lin}^{-} \mathrm{Sca}-1^{+} \mathrm{c}-\mathrm{kit}^{+}(\mathrm{LSK})$

6 HSCs were seeded with $\operatorname{Lin}^{+}$niche cells over a range of HSC:Lin ${ }^{+}$densities (1:0, 1:10, 1:100).

7 All experimental conditions contained an identical number of HSCs (5000). Overall viability of

8 the HSC:Lin ${ }^{+}$cell co-cultures (1:0 to 1:100) was high (>90\%) for all conditions (Fig. 2A). Flow

9 cytometry analysis revealed that after two days of co-culture, the total number of LSK cells

10 expanded (Fig. 2B) with increasing niche cell density. Further, early hematopoietic progenitors,

11 long-term HSCs (LT-HSCs; LSK·CD34 ${ }^{\mathrm{lo}} \cdot \mathrm{Flk} 2^{\mathrm{lo} /}$ ), short-term HSCs (ST-HSCs;

$12 \mathrm{LSK} \cdot \mathrm{CD} 34^{\mathrm{hi}} \cdot \mathrm{Flk} 2^{\mathrm{lo} /}$ ), and multipotent progenitors (MPPs; LSK $\cdot \mathrm{CD} 34^{\mathrm{hi}} \cdot \mathrm{Flk} 2^{\mathrm{hi}}$ ) showed

13 significant increases with increasing $\operatorname{Lin}^{+}$niche cell density (Fig. 2C-E). Effects of Lin ${ }^{+}$co-

14 culture on myeloid differentiation was also seen, with common myeloid progenitors (CMP; Lin

$15 \cdot \mathrm{IL} 7 \mathrm{R} \alpha^{-} \cdot \mathrm{c}-\mathrm{kit}^{+} \cdot \mathrm{Sca}-1^{-} \cdot \mathrm{CD} 34^{+} \cdot \mathrm{CD} 16 / 32^{-}$) showing a non-significant decrease with $\mathrm{Lin}^{+}$density

16 and myeloid erythroid progenitors (MEP; $\left.\mathrm{Lin}^{-} \cdot \mathrm{IL} 7 \mathrm{R} \alpha^{-} \cdot \mathrm{c}-\mathrm{kit}^{+} \cdot \mathrm{Sca}-1^{-} \cdot \mathrm{CD} 34^{-} \cdot \mathrm{CD} 16 / 32^{-}\right)$showing a

17 significant increase with $\mathrm{Lin}^{+}$density. No effect was observed for granulocyte macrophage

18 progenitors (GMP; $\left.\operatorname{Lin}^{-} \cdot \mathrm{IL}_{\mathrm{R}} \alpha^{-} \cdot \mathrm{c}-\mathrm{kit}^{+} \cdot \mathrm{Sca}-1^{-} \cdot \mathrm{CD} 34^{+} \cdot \mathrm{CD} 16 / 32^{+}\right)($Fig. 2F-H). Colony forming

19 unit (CFU) assays revealed minor functional changes as well. Notably, a decrease in the most

20 primitive GEMM (granulocyte, erythrocyte, macrophage, ) colonies, a significant $(p<0.01)$

21 increase in CFU-M (macrophage) colonies, and increasing trends in granulocyte macrophage

22 (GM) and granulocyte (G) colonies (Supplementary Fig. 1). These results suggest that co- 


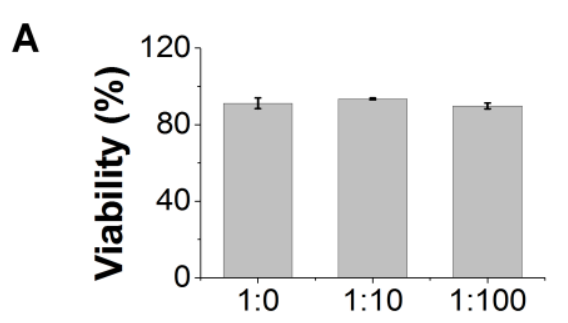

LSK:Lin ${ }^{+}$cell ratio

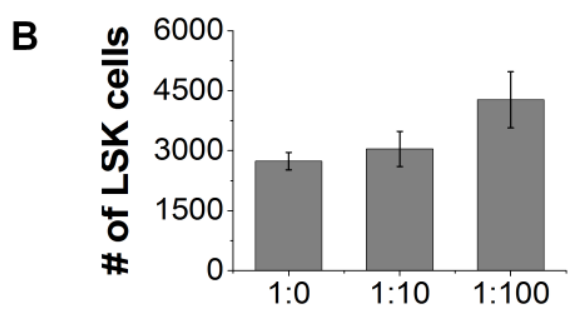

LSK:Lin ${ }^{+}$cell ratio
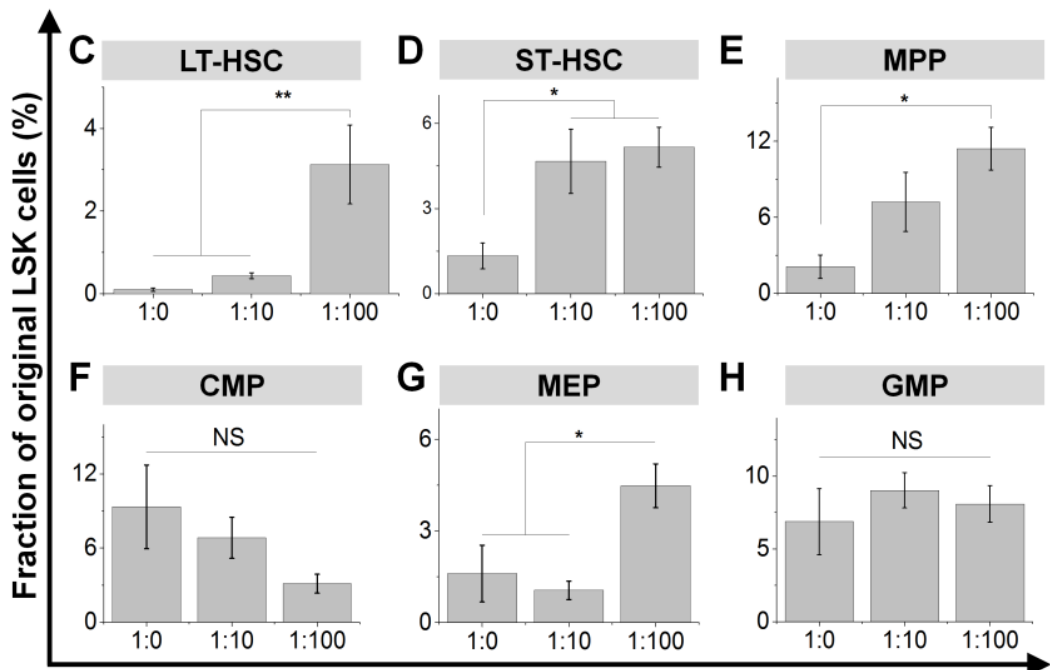

LSK:Lin ${ }^{+}$cell ratio

Figure 2. Co-culture of LSK cells with Lin+ niche cells in liquid (2D) culture.

(A) Viability of Lin-Sca1 ${ }^{+} \mathrm{CKit}^{+}$(LSK) HSC as a function of niche cell density. (B) Total number of LSK cells remaining as a function of niche cell density. (C) - (H) Fraction of the early and late hematopoietic progenitors as a function of LSK:niche cell co-culture density. LT-HSC: Long-term repopulating HSC (LSK·CD34-Flk2 $\left.{ }^{-}\right)$. ST-HSC: Short-term repopulating HSC. MPP: Multipotent progenitor (LSK·CD34-Flk2'). CMP: common myeloid progenitor. MEP: megakaryocyte-erythrocyte progenitor. GMP: granulocyte-macrophage progenitor. ${ }^{*}: p<0.05 .{ }^{* *}: p<0.01 .{ }^{* * *}: p<0.005$. NS: No significance.

culture with $\mathrm{Lin}^{+}$niche cells altered LSK proliferation and lineage specification, with

2 particularly significant effects on early progenitor fractions.

\subsection{Biophysical characterization of collagen hydrogels}

5 The storage modulus $\left(G^{\prime}(\omega)\right)$ for both collagen hydrogel concentrations $(1 \mathrm{mg} / \mathrm{ml}, 3 \mathrm{mg} / \mathrm{ml})$ was significantly larger than the viscous modulus $\left(G^{\prime \prime}(\omega)\right)$, suggesting the materials are effectively

7 solid over the timescales (frequencies: $10^{-1}-10^{2} \mathrm{rad} / \mathrm{sec}$ ) probed. We report the plateau modulus 
1 modulus of $5 \mathrm{~Pa}$, while the $3 \mathrm{mg} / \mathrm{ml}$ collagen hydrogel exhibited viscoelastic behavior and a

2 plateau modulus of $56 \mathrm{~Pa}$. These rheological properties were also used to estimate changes in

3 network mesh size $\xi$ with collagen concentration. With fiber diameter $d$, we use the relation $G_{0}$

$4 \sim \xi^{-22 / 5} d^{28 / 5}$ from an existing theory for semi- flexible polymer networks [31, 32], suggesting a

$540 \%$ decrease in mesh size for 3 vs. $1 \mathrm{mg} / \mathrm{ml}$ gels. Data at high frequency is affected by

6 instrument rotational inertia, and cannot be used for analysis (dashed line) (Supplementary Fig.

7 2A) [39]. Longer timescales were probed with step-stress (creep) tests at an input stress of 0.03

$8 \mathrm{~Pa}$ (Supplementary Fig. 2b). Up to timescales of 100s, both materials show solid-like behavior

9 (plateaus of the creep compliance, $J(t)$ ), with time. Upon close inspection (Supplementary Fig.

$102 \mathrm{~b}$ inset), the $1 \mathrm{mg} / \mathrm{ml}$ collagen gel exhibits slight power law creep compliance

$11 J(t) \approx\left[0.15 \frac{1}{\mathrm{~Pa}^{0.1}}\right] t^{0.1}$, suggesting soft solid behavior close to a critical gel [40].

13 Gel diffusivity indicates collagen concentration and molecular size dependent effects on

14 diffusivity. The diffusivity of $40 \mathrm{kDa}$ Dextran decreased significantly with increasing hydrogel

15 density ( $\left.1 \mathrm{mg} / \mathrm{mL}: 15.9 \pm 0.67 \mu \mathrm{m}^{2} / \mathrm{sec} ; 3 \mathrm{mg} / \mathrm{mL}: 10.7 \pm 0.67 \mu \mathrm{m}^{2} / \mathrm{sec}\right)($ Supplementary Fig.

16 2C). Diffusivity of $2000 \mathrm{kDa}$ Dextran was significantly lower for both hydrogel formulations,

17 but was insensitive to collagen concentration, likely due to a larger hydrodynamic diameter $\left(d_{h} \sim\right.$

$1870 \mathrm{~nm}$ ) on order of the collagen mesh size. For $40 \mathrm{kDa}$ molecules, the hydrodynamic diameter $d h$

$19 \sim 8 \mathrm{~nm}$ is much smaller than the collagen mesh size [33, 41]. As many hematopoietic associated

20 cytokines (e.g., SCF [10], CXCL12, VEGF, TGF $\beta$ ) range in size between $20-60 \mathrm{kDa}$, these two

21 hydrogel variants present significantly different biotransport environments for paracrine or

22 autocrine-mediated signals. 
1 Cell spacing for each niche cell condition was calculated assuming a simple cubic lattice

2 structure of cell arrangement in the 3D hydrogels. The characteristic diffusion time between cells

3 ( $\left.t_{D}\right)$ was calculated based on these distances and the experimentally determined diffusion

4 coefficient (D) according to (Supplementary Table 1):

5

6

22 Heat maps normalizing cell response of HSC:niche co-culture in the hydrogel network versus

23 liquid co-cultures were used to compare the magnitude and effect of the presence of a hydrogel

\subsection{HSC progenitor response to $\mathrm{Lin}^{+}$niche cells is altered by the surrounding hydrogel}

\section{network}

Early progenitor fractions (LT-HSC, ST-HSC, MPP) showed significant $(p<0.05)$ increases with increasing $\mathrm{Lin}^{+}$co-culture in $1 \mathrm{mg} / \mathrm{mL}$ gels. While the trend still existed in $3 \mathrm{mg} / \mathrm{mL}$ hydrogels, the differences were no longer significant (Fig. 3A). Looking at differentiating hematopoietic populations, the number of common myeloid progenitors (CMPs) held constant for increasing $\mathrm{Lin}^{+}$niche cells, while the further differentiated myeloid progenitors (MEP, GMP) showed significant increases $\left(p<0.01\right.$ for $1 \mathrm{mg} / \mathrm{ml} ; p<0.05$ for $3 \mathrm{mg} / \mathrm{ml}$ ) with $\mathrm{Lin}^{+}$co-culture (Supplementary Fig. 3B). Analysis of the functional capacity of the HSCs via CFU assays revealed similar conclusions, notably an increase in downstream myeloid progenitors (GM, G, M and E colonies) in both $1 \mathrm{mg} / \mathrm{ml}$ and $3 \mathrm{mg} / \mathrm{ml}$ collagen gels (Supplementary Fig. 4, 5).

Although not statistically significant, while numbers of the most primitive GEMM colonies decreased with increasing $\mathrm{Lin}^{+}$co-culture in liquid media, GEMM colonies remained relatively constant or increased in hydrogel cultures.

$$
t_{D}(s)=\frac{\text { Cell spacing }{ }^{2}\left(\mu m^{2}\right)}{D\left(\mu m^{2} / s\right)}
$$



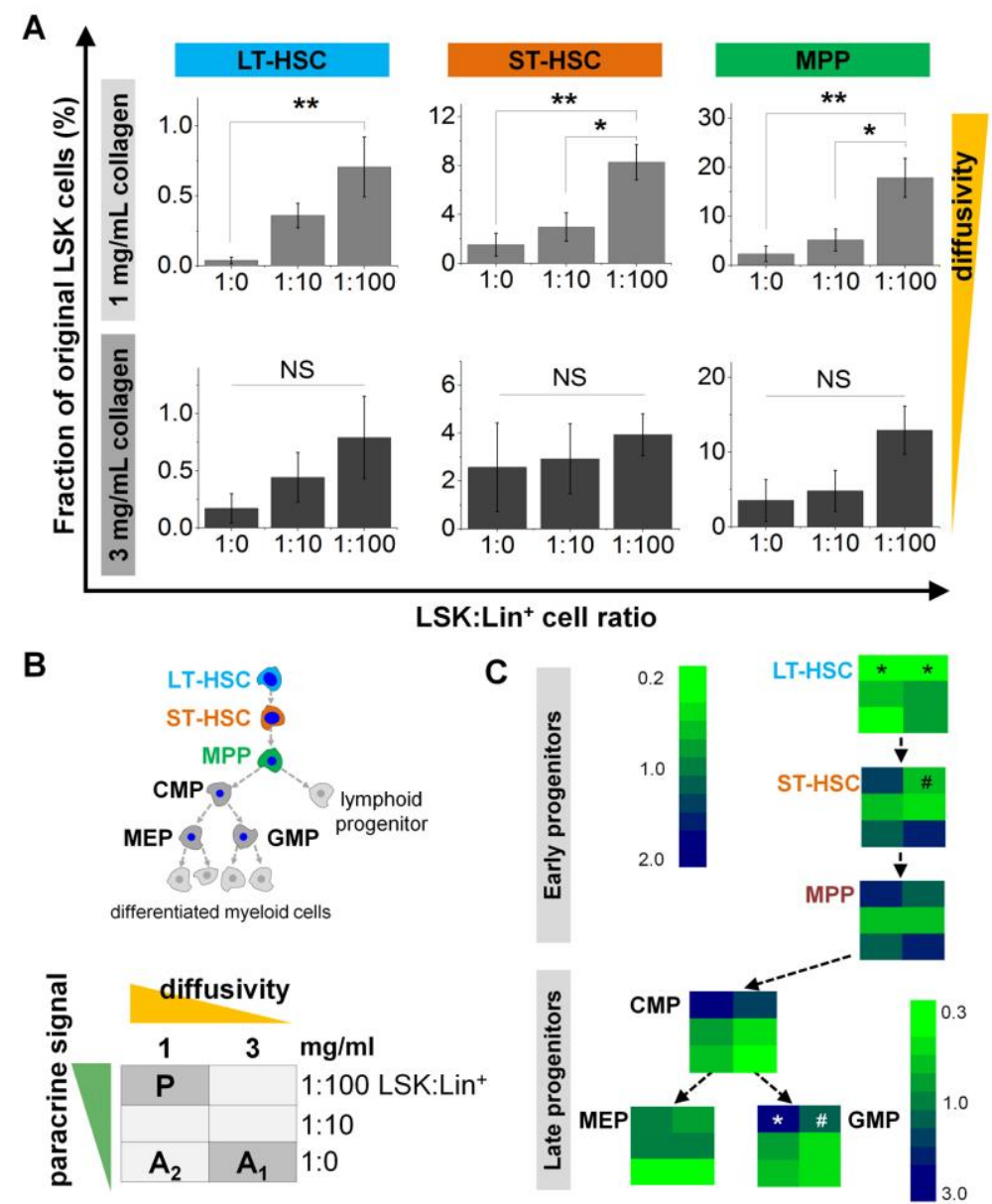

Figure 3. Comparative analysis of hematopoietic progenitor populations via flow cytometry as a function of hydrogel matrix conditions and niche cell co-culture density.

(A) Early hematopoietic progenitor populations (LT-HSC, ST-HAS, MPP) increase with increasing niche cell density. (B) Representative schematic of the HSC differentiation hierarchy and heat map legend depicting changes in HSC:niche cell density ('paracrine signal) as well as hydrogel density ('diffusivity'). (C) Heat map depicting relative changes in progenitor cell populations in 1 and $3 \mathrm{mg} / \mathrm{mL}$ collagen hydrogel conditions normalized against observed changes in 2D liquid (diffusion-unlimited) culture. Separate heat map scales are used for Early vs. Late hematopoietic progenitors to better demonstrate effects of niche cell and hydrogel density. ${ }^{*}: p<0.05$ w.r.t. $2 D$ culture. ${ }^{*}$ : $p<0.01$ w.r.t. $2 D$ culture. ${ }^{* * *}: p<0.001$ w.r.t. $2 D$ culture. $\#: p<0.05$ w.r.t. $1 \mathrm{mg} / \mathrm{mL}$ culture.

1 network on HSC differentiation. For each cell state (e.g., CD34-Flk2-LSK LT-HSCs), a $3 \times 2$

2 heat map compares 6 microenvironmental conditions (Fig. 3B). Rows correspond to discrete

3 HSC:Lin ${ }^{+}$cell densities (1:0, bottom; 1:10; 1:100 top) while columns correspond to hydrogel

4 density $(1 \mathrm{mg} / \mathrm{ml}$ left; $3 \mathrm{mg} / \mathrm{ml}$ right). Therefore, the bottom right of each matrix corresponds to a

5 niche environment with no niche cells and increased restriction to biomolecule diffusion

6 (maximum autocrine feedback). The top left corner (high niche cell:HSC density; high 
1 diffusivity) corresponds to the highest potential paracrine signal environment. Relative increases

2 in early progenitor fractions (LT-HSC, ST-HSC) were observed for autocrine feedback

3 dominated (3 mg/ml, 1:0 HSC:Lin ${ }^{+}$ratio). However the greatest increase in late-progenitor cell

4 populations (CMP, GMP) was observed in paracrine-dominated regimes $(1 \mathrm{mg} / \mathrm{mL}$ hydrogel;

5 1:100 HSC:Lin ${ }^{+}$ratio) (Fig. 3B). Heat maps regarding functional CFU formation were consistent

6 with FACS results, suggesting that except for GEMM colonies, lower diffusivity ( $3 \mathrm{mg} / \mathrm{mL}$ )

7 hydrogels resulted in lower colony numbers than higher diffusivity $(1 \mathrm{mg} / \mathrm{mL})$ hydrogels

8 (Supplementary Fig. 6). Taken together, these results suggest that reduced gel diffusivity

9 promotes expansion of early HSC progenitors at low niche cell densities, whereas conditions of

10 high niche cell density and increased gel diffusivity promote expansion of differentiated

11 hematopoietic progenitors.

\section{$13 \quad 3.4$ Inhibition of paracrine signals affects hematopoietic progenitors at all levels}

14 To better describe the effect of paracrine signals from $\mathrm{Lin}^{+}$niche cells on HSC response, cells

15 were again co-cultured in the $1 \mathrm{mg} / \mathrm{ml}$ collagen hydrogel (high diffusivity) in the presence of a

16 cocktail of inhibitors for TGF- $\beta$, MIP- $1 \alpha$ and TNF $\alpha$ (Fig. 4A), paracrine signals known to inhibit

17 the self-renewal capacity of primitive HSC progenitor populations [23]. Here, heat maps

18 comparing HSC response (relative to liquid culture) contain rows for increasing $\operatorname{Lin}^{+}$density as

19 before, but columns now compare uninhibited (left) vs. paracrine signal inhibited (right) culture

20 (Fig. 4B). While dependence of early HSC progenitors on niche cell density was qualitatively

21 similar for the paracrine signal inhibited conditions, we observed a reduction in differentiated

22 myeloid populations. The greatest effect was seen for late myeloid progenitors (CMP, GMP) at

23 high niche cell densities. Compared to uninhibited cultures, early progenitor (e.g., CMP) 

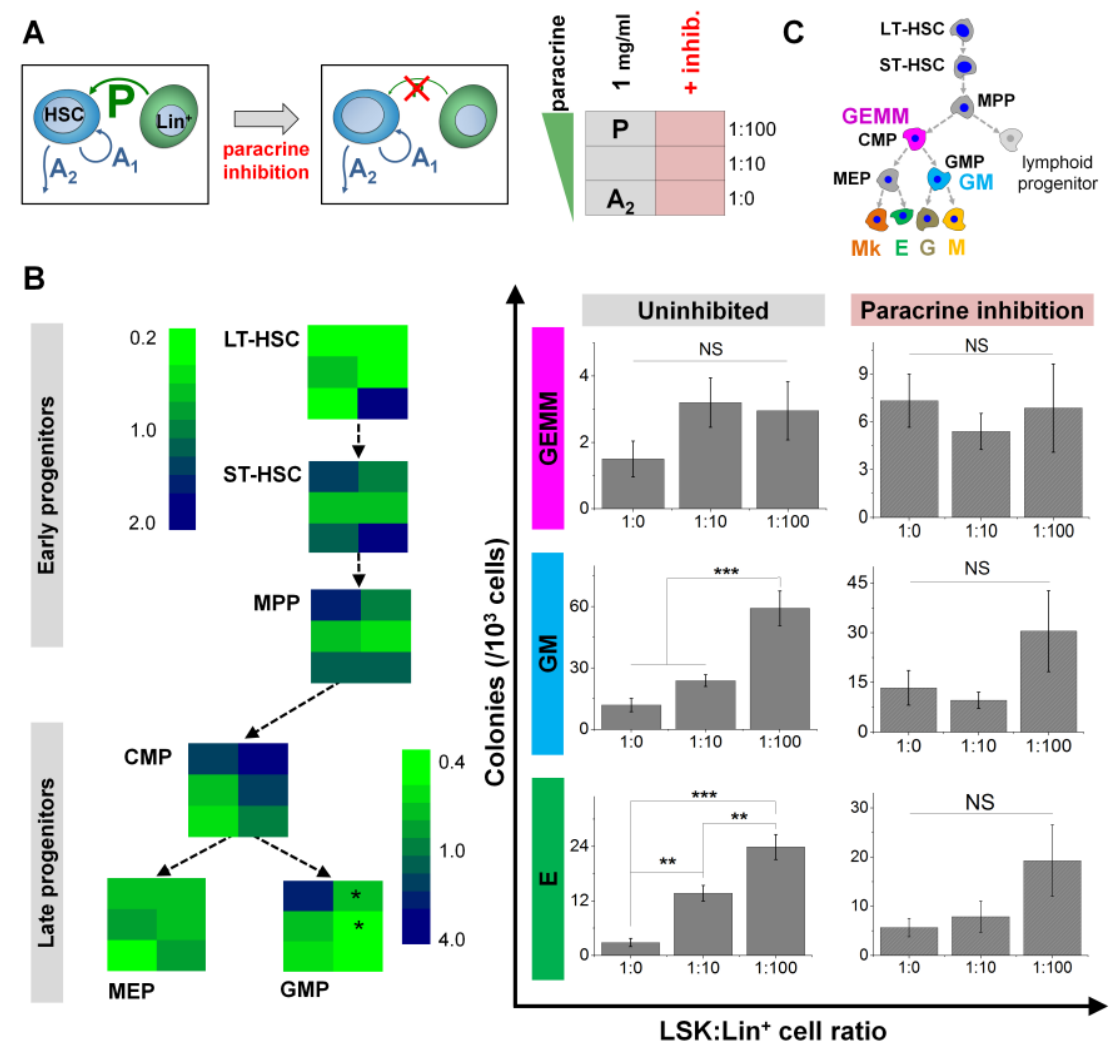

Figure 4. Effect of the addition of paracrine-inhibitory cocktail on HSC response to Lin+ niche cells within the hydrogel environment.

(A) A schematic depicting the effect of paracrine inhibition on HSCs in $1 \mathrm{mg} / \mathrm{mL}$ collagen co-cultures. HSC response was quantified via surface antigen expression using a modified heat map depiction of the effects of paracrine inhibition (right column) vs. culture in $1 \mathrm{mg} / \mathrm{ml}$ collagen (left column).

Functional changes in HSC response were also profiled via CFU-assays (CFU-GEMM, CFU-GM, along with more mature Mk, E, G, M colonies). (B) Heat map-based analysis of effect of addition of paracrine inhibitor cocktails to HSC:Lin niche cell co-culture in paracrine-dominated $(1 \mathrm{mg} / \mathrm{mL})$ hydrogel environments showing fold change in cell fractions both without (left column) and with (right column) paracrine inhibitors, with values normalized against 2D co-culture. (C) CFU assay showing effect of paracrine inhibition on progressively mature colonies, GEMM (most primitive) to $E$ (most mature). ${ }^{*}: p<0.05$ w.r.t. 2D culture. ${ }^{* *}: p<0.01$ w.r.t. 2D culture. ${ }^{* * *}: p<0.001$ w.r.t. 2D culture. NS: Not significant.

fractions increased whereas the more mature GMP fractions decreased. Results from CFU assays

exhibited a similar trend. While no significant changes were seen in the primitive GEMM

3 colonies due to loss of paracrine signaling, more mature colonies such as GM and E no longer showed a significant increase in number with $\operatorname{Lin}^{+}$niche cell density (Fig. 4C, Supplementary

Fig. 8). 
1 We subsequently co-cultured HSCs in $3 \mathrm{mg} / \mathrm{mL}$ collagen (low diffusivity) hydrogels in the

2 presence of a cocktail of inhibitors for VEGF, PDGF and FGF receptors (Fig. 5A). Autocrine

3 mediated feedback via these factors has been shown to promote primitive HSC expansion [17,

$423,42]$, suggesting their inhibition in hydrogel culture may reduce the numbers of early

5 hematopoietic progenitors in conditions where autocrine feedback is significant (low niche cell

6 densities; low diffusivity environments). Heat maps comparing HSC response (relative to liquid

7 culture) contain rows for increasing $\operatorname{Lin}^{+}$density as before, but columns now compare
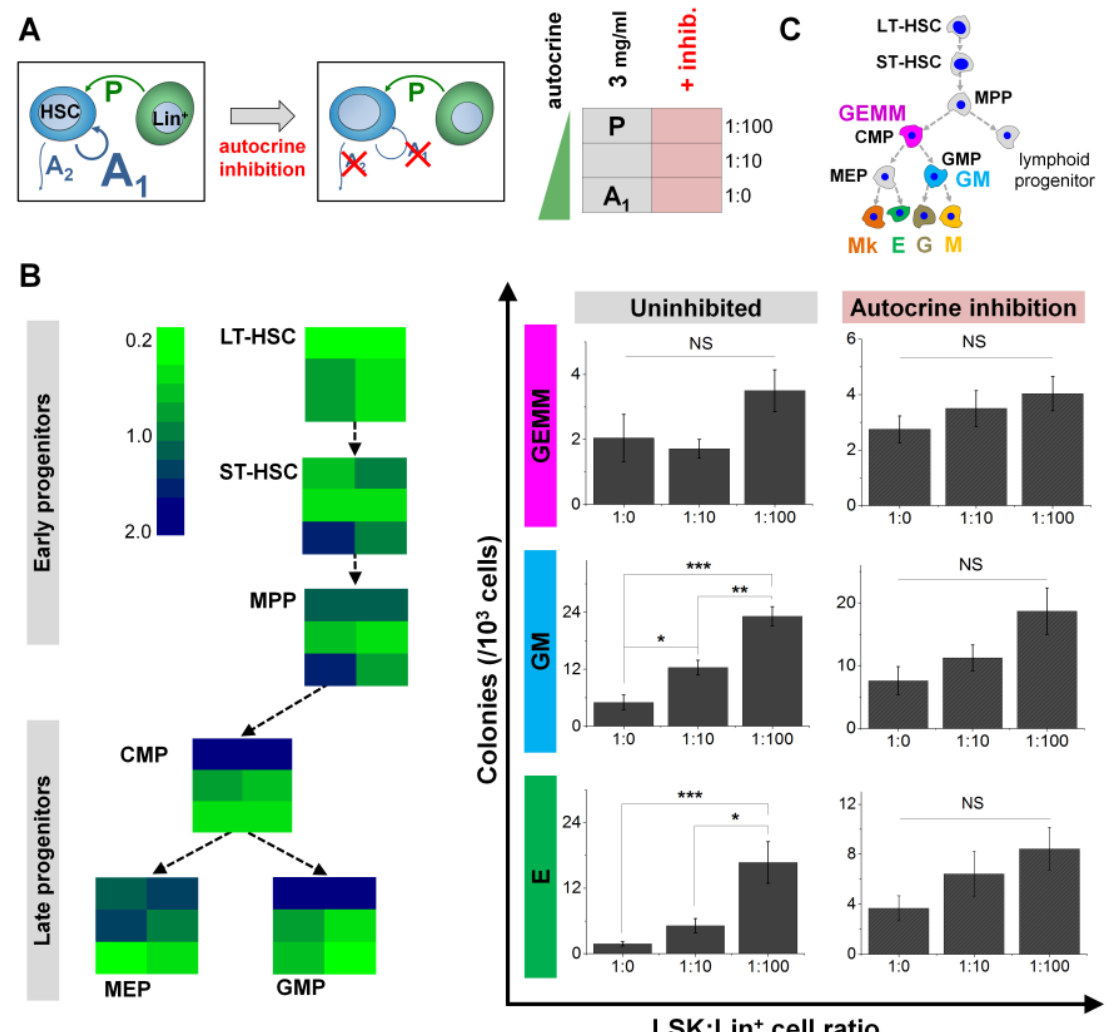

Figure 5. Effect of the addition of autocrine-inhibitors on HSC response within the hydrogel environment.

(A) A schematic hypothesizing the effects of autocrine inhibition in $3 \mathrm{mg} / \mathrm{mL}$ collagen co-cultures. HSC response was quantified via surface antigen expression using a modified heat map depiction of the effects of autocrine inhibition (right column) vs. culture in $3 \mathrm{mg} / \mathrm{ml}$ collagen (left column). Functional changes in HSC response were also profiled via CFU-assays (CFU-GEMM, CFU-GM, along with more mature Mk, E, G, M colonies). (B) Heat map-based analysis of effect of addition of autocrine inhibitor cocktails to HSC:Lin ${ }^{+}$niche cell co-culture in autocrine-dominated $(3 \mathrm{mg} / \mathrm{mL})$ hydrogel environments showing fold change in cell fractions both without (left column) and with (right column) autocrine inhibitors, with values normalized against 2D co-culture. (C) CFU assay showing effect of paracrine inhibition on progressively mature colonies, GEMM (most primitive) to $E$ (most mature). *: $p$ $<0.05$ w.r.t. 2D culture. ${ }^{* *}: p<0.01$ w.r.t. $2 D$ culture. ${ }^{* * *}: p<0.001$ w.r.t. $2 D$ culture. NS: Not significant. 
1 uninhibited (left) vs. autocrine signal inhibited (right) culture (Fig. 5B). Interestingly, there was a

2 reduction in the fold change in early progenitor populations, with the greatest reductions seen for

3 the ST-HSC and MPP fractions at low (1:0) niche cell densities as per our hypothesis. Further,

4 the non-significant reduction in the reduced number of LT-HSCs and ST-HSCs in conventional

$53 \mathrm{mg} / \mathrm{ml}$ culture with decreasing niche cell density (1:0 vs. 1:100; Fig. $3 \mathrm{~A}, 3 \mathrm{mg} / \mathrm{ml}$ condition)

6 became a significant reduction in the presence of autocrine inhibition $(p<0.005)$

7 (Supplementary Fig. 9). These findings are similar to the significant reduction observed in non-

8 autocrine inhibited high-diffusivity $(1 \mathrm{mg} / \mathrm{ml}$ ) hydrogels (Fig. 3A, $1 \mathrm{mg} / \mathrm{ml}$ condition). Despite

9 these changes in early hematopoietic progenitor populations, numbers of late progenitor fractions

10 (e.g., CMP, MEP, GMP; Fig. 5B, Supplementary Fig. 9) as well as colony forming unit assays

11 (GM, G, M, Mk, E; Supplementary Fig. 10) remained relatively unchanged.

\section{4. Discussion}

14 Signaling molecules play a central role in regulating stem cell fate in the in vivo HSC niche.

15 Signaling pathways associated with many biomolecules have been evaluated in the context of

16 activated paracrine or autocrine signaling channels. However these interactions have not been

17 translated to 3D culture systems. One main challenge is the interdependence of stiffness,

18 diffusivity, and ligand density. Matrix stiffness is a function of the crosslinking density, which

19 also controls small molecule diffusion through its fiber network with higher stiffness

20 corresponding to lower diffusion [43]. Although decoupling matrix stiffness and diffusion effects

21 is difficult, proper selection of gel properties can help mitigate their biological effects. Discher $e t$

22 al. showed CD34 ${ }^{+}$HSCs exhibited different morphologies when cultured on substrates with a

$23100 x$ difference in stiffness $(0.3 \mathrm{kPa}$ vs $34 \mathrm{kPa})$ [44]. However, Choi et al. demonstrated that 
1 while HSC bioactivity was impacted by substrate elasticity between $1 \mathrm{kPa}$ to $196 \mathrm{kPa}$, no

2 significant dependence was observed for softer substrates ( $<100 \mathrm{~Pa})$ [11]. The collagen gel

3 stiffness explored here falls well below these ranges (5.5 Pa, $1 \mathrm{mg} / \mathrm{mL} ; 56 \mathrm{~Pa}, 3 \mathrm{mg} / \mathrm{mL}$ ),

4 suggesting a reduce role of matrix stiffness on HSC response in these studies. The influence of

5 ligand density, which increases with collagen density ( $1 \mathrm{mg} / \mathrm{mL}$ vs. $3 \mathrm{mg} / \mathrm{mL})$, on HSC behavior

6 has also been previously documented $[11,45,46]$; while the collagen hydrogel system employed

7 here precludes close control over changes in ligand density, the marked effects of niche cell co-

8 culture as well as paracrine and autocrine inhibition on HSC response observed here suggest an

9 opportunity to design improved biomaterials for selective HSC expansion. Though the

10 importance of niche cell populations on HSC fate decisions in vitro is well known, most previous

11 works focus on direct cell-cell contact $[47,48]$ or the effects of paracrine signals in liquid culture

12 [25]. Through the use of defined 3D hydrogel matrices, we report HSC expansion and myeloid

13 differentiation can be significantly altered by feedback-dependent effects of $\mathrm{Lin}^{+}$niche cells and

14 HSCs themselves.

16 Our results reveal the presence of a hydrogel matrix altered the signaling feedback between $\mathrm{Lin}^{+}$

17 niche cells (paracrine) and LSK generated (autocrine) feedback. The $3 \mathrm{mg} / \mathrm{mL}$ collagen hydrogel

18 environment promoted a higher fraction of the early progenitor fractions at low niche cell

19 densities (Fig. 3C). While reducing the number of $\operatorname{Lin}^{+}$niche cells $(1: 100,1: 10,1: 0)$ led to a

20 significant reduction in LT-HSC, ST-HSC, and MPP in liquid culture and more diffusive $1 \mathrm{mg} / \mathrm{ml}$

21 hydrogels (Fig. 3A), the reduction was not significant for the $3 \mathrm{mg} / \mathrm{ml}$ hydrogel, suggesting the

22 importance of autocrine feedback in dictating primitive HSC fate decisions. Similarly, the 
1 increased incidence of late progenitors in the $1 \mathrm{mg} / \mathrm{mL}$ gels at high niche cell densities suggest

2 the dominance of paracrine signals.

4 We next asked whether the introduction of inhibitor cocktails for known paracrine signaling

5 paths could abrogate effects observed at high niche cell and high matrix diffusivity conditions.

6 Coincident increases in LT-HSC and ST-HSC cell populations, significant reduction in

7 downstream granulocyte-macrophage progenitors (GMPs) (Fig. 4B), and reduced numbers of

8 downstream CFU-GM and E colonies is consistent with this hypothesis (Fig. 4C). Tellingly, the

9 greatest changes in the late progenitor fractions with paracrine signal inhibition were seen in the

10 highest niche cell density cultures (1:100). This suggests the exciting potential to use expanded

11 time-resolved analyses to examine the impact of paracrine-autocrine signals in future studies.

12 Similarly, inhibition of autocrine signaling pathways, specifically VEGF, PDGF and FGF,

13 altered enhanced early hematopoietic progenitor populations in diffusion-limited or low niche

14 cell density hydrogels (Fig. 5A). While late progenitor fractions were not affected by autocrine

15 inhibition, early progenitor fractions were reduced, particularly in the case of HSC only (1:0)

16 cultures (Fig. 5B). Hence, disruptions to autocrine feedback via the hydrogel environment may

17 be particularly important for altering expansion of early hematopoietic progenitors. Together,

18 these observations raise a number of exciting questions for future study to investigate dose-

19 dependent effects of singular inhibitors and cocktails, expand the range of hydrogel

20 environments considered, and explore the effects of matrix-remodeling based dynamic changes

21 in biotransport. 
1 These observations provide a perspective on the characteristic diffusion time $\left(t_{D}\right)$ of autocrine

2 and paracrine signaling molecules. Given the complex nature of co-culture conditions and the

3 inter-dependent factors influencing cell biology, $t_{D}$ provides a tunable property capable of

4 decoupling some of these effects. For example, despite only a 1.5 -fold difference in matrix

5 diffusivity for the 1 vs. $3 \mathrm{mg} / \mathrm{mL}$ collagen hydrogel, vastly different HSC biology was observed

6 even at similar cell concentrations. Characteristic diffusion times on the order of $4400 \mathrm{~s}(3 \mathrm{mg} / \mathrm{mL}$

7 hydrogel) were sufficient to promote autocrine signal-mediated expansion of early HSC

8 progenitors. Interestingly, characteristic $t_{D}$ of order $3000 \mathrm{~s}(1 \mathrm{mg} / \mathrm{mL}$ hydrogel $)$ did not show the

9 same effect for HSC only cultures, while $t_{D}$ of order of 540s in the presence of $\mathrm{Lin}^{+}$niche cells

10 was sufficient to promote paracrine signal mediated hematopoietic differentiation. These

11 findings are conceptually consistent with those recently reported by Müller et al. for culture of

12 HSPCs in media filled microcavities; autocrine feedback promoted HSC quiescence but could

13 also be abrogated by expanding the size of the microcavity to dilute the effect [26]. In our work,

14 increasing the characteristic $t_{D}$ of fully three-dimensional hydrogels was able to promote

15 autocrine-mediated HSC expansion, suggesting selective engineering of characteristic diffusion

16 times provides an important avenue for hematopoietic stem cell niche engineering in fully three-

17 dimensional biomaterial environments.

19 The ability to concentrate the design of hydrogel networks to alter autocrine/paracrine feedback

20 in heterogeneous niche-stem cell cultures offers an exciting possibility in biomaterial design. The

21 well-defined biological underpinnings of the HSC system [49], along with significant in vivo

22 data regarding multiple niche cell populations of potential functional importance motivate this

23 investigation [50]. A broad spectrum of conditions, both in niche cell and matrix concentration, 
1 was chosen to obtain a comprehensive perspective of HSC differentiation trends in vitro.

2 Although the variables under study are multi-faceted and co-dependent to draw pointed

3 conclusions, the relative effects and trends observed here suggest engineering a system that can

4 explicitly define these effects will be instrumental for future HSC biomanufacturing applications.

5 It is likely identification of culture conditions to expand early hematopoietic progenitors will

6 require increased resolution of the effect of discrete signaling pathways on HSC fate and whether

7 singular or coordinated inhibition of these pathways can selectively enhance the effect. Further,

8 while we were able to examine a broad spectrum of cell density $(1: 0-1: 100)$, the range of

9 hydrogel environments tested here, while sufficient to resolve important differences in paracrine

10 signals vs. autocrine feedback, represents only a small fraction of available design space.

11 Ongoing efforts are using a methacrylamide-functionalized gelatin hydrogel system that is

12 compatible with analysis of HSC differentiation patterns [10] and provides the capability of

13 generating libraries of hydrogel biomaterials with tunable matrix properties [29, 51]. Further, this

14 study chose to use a Flk-based FACS sorting paradigm as well as CFU assays that allowed us to

15 parse early hematopoietic progenitor populations as LT-HSC vs. ST-HSC vs. MPP. While

16 chosen to allow us to more broadly subtype HSC response to niche cell co-culture, future efforts

17 will focus on more stringent identification of LT-HSCs via surface antigen markers (e.g., Lin

$18 \mathrm{Sca}^{+} \mathrm{CKit}^{+} \mathrm{CD} 150^{+} \mathrm{CD} 48^{-\mathrm{CD}} 244$ [52] and in vivo transplantation assays [53]. Finally, the choice

19 of niche cell type provides an opportunity to explore differences in paracrine signals. While $\mathrm{Lin}^{+}$

20 niche cells comprise a broad spectrum of cell populations with a heterogeneous biomolecule

21 profile, incorporation of discrete quiescent versus active niche inspired cell populations such as

22 mesenchymal stem cells or vascular endothelial cells will help accurately classify the role and

23 mechanisms of the biomolecules involved in autocrine and paracrine pathways at different stages 
1 of lineage specification. The overarching objective of this study was to address the critical

2 question of how signaling mechanisms within a complex 3D co-culture environment are

3 influenced by surrounding biophysical and biochemical properties and their resulting effect on

4 HSC biology, and our observations provide a concrete direction for future studies addressing the

5 interplay of HSC - matrix - biomolecule synergy.

\section{5. Conclusions}

8 Interactions between HSCs and niche cells within the bone marrow involve activation of

9 autocrine, paracrine and juxtacrine-contact associated signaling pathways. The nature of these

10 interactions, both complex and dynamic, motivates the design of ex vivo culture systems to

11 explore the nature of these interactions. Here we describe an approach co-varying hydrogel

12 diffusivity and niche cell density as a means to alter early HSCs fate decisions. We show that in

13 paracrine-signaling dominated hydrogel regimes, the use of $\mathrm{Lin}^{+}$niche cells can enhance HSC

14 myeloid lineage specification while these effects can be abrogated via inhibitory cocktails of

15 paracrine signaling molecules. Autocrine-dominated regimes, characterized by low diffusivity

16 hydrogel environments, can both insulate HSC response from niche cell co-culture but

17 potentially also enhance the effect of HSC autocrine feedback events. These effects could be

18 reduced by selective inhibition of autocrine signaling molecules. Together, these results suggest

19 the exciting possibility to exploit the balance of paracrine and autocrine signals through selective

20 design of three-dimensional culture tools to gain improved control of HSC fate in vitro.

\section{ACKNOWLEDGEMENTS}


1 The authors would like to acknowledge Barbara Pilas and Bill Hanafin (Flow Cytometry

2 Facility, UIUC) as well as Ms. Sunny Choi (ChBE, UIUC) for assistance with bone marrow cell

3 isolation and flow cytometry. This work was partially supported by Grants \#160673 and

$4 \quad \# 189782$ from the American Cancer Society, Illinois Division, Inc. This material is based upon

5 work supported by the National Science Foundation under Grant No. 1254738 (B.A.C.H.).

6 Research reported in this publication was supported by the National Institute of Diabetes and

7 Digestive and Kidney Diseases of the National Institutes of Health under Award Number R01

8 DK099528 (B.A.C.H). The authors are also grateful for additional funding provided by the

9 Department of Chemical \& Biomolecular Engineering and the Institute for Genomic Biology at 10 the University of Illinois at Urbana-Champaign. 


\section{References}

[1] R. Schofield, The relationship between the spleen colony-forming cell and the haemopoietic stem cell, Blood cells 4(1-2) (1978) 7-25.

[2] D.N. Haylock, S.K. Nilsson, Stem cell regulation by the hematopoietic stem cell niche, Cell Cycle 4(10) (2005) 1353-5.

[3] K. Tokoyoda, T. Egawa, T. Sugiyama, B.I. Choi, T. Nagasawa, Cellular niches controlling B lymphocyte behavior within bone marrow during development, Immunity 20(6) (2004) 707-18. [4] L.E. Purton, D.T. Scadden, The hematopoietic stem cell niche, StemBook, Cambridge (MA), 2008.

[5] G.B. Adams, D.T. Scadden, The hematopoietic stem cell in its place, Nat Immunol 7(4) (2006) 333-7.

[6] D.T. Scadden, The stem-cell niche as an entity of action, Nature 441(7097) (2006) 10751079 .

[7] A. Wilson, A. Trumpp, Bone-marrow haematopoietic-stem-cell niches, Nat Rev Immunol 6(2) (2006) 93-106.

[8] J. Isern, S. Mendez-Ferrer, Stem cell interactions in a bone marrow niche, Current osteoporosis reports 9(4) (2011) 210-8.

[9] M.V. Gomez-Gaviro, R. Lovell-Badge, F. Fernandez-Aviles, E. Lara-Pezzi, The Vascular Stem Cell Niche, Journal of cardiovascular translational research (2012).

[10] B.P. Mahadik, S. Pedron Haba, L.J. Skertich, B.A. Harley, The use of covalently immobilized stem cell factor to selectively affect hematopoietic stem cell activity within a gelatin hydrogel, Biomaterials 67 (2015) 297-307.

[11] J.S. Choi, B.A. Harley, The combined influence of substrate elasticity and ligand density on the viability and biophysical properties of hematopoietic stem and progenitor cells, Biomaterials 33(18) (2012) 4460-8.

[12] J.E. Nichols, J. Cortiella, J. Lee, J.A. Niles, M. Cuddihy, S. Wang, J. Bielitzki, A. Cantu, R. Mlcak, E. Valdivia, R. Yancy, M.L. McClure, N.A. Kotov, In vitro analog of human bone marrow from 3D scaffolds with biomimetic inverted colloidal crystal geometry, Biomaterials 30(6) (2009) 1071-9.

[13] M.L. Cuchiara, S. Coşkun, O.A. Banda, K.L. Horter, K.K. Hirschi, J.L. West, Bioactive poly(ethylene glycol) hydrogels to recapitulate the HSC niche and facilitate HSC expansion in culture, Biotechnol Bioeng 113(4) (2016) 870-881.

[14] Y. Tajima, E.J. Huang, K. Vosseller, M. Ono, M.A. Moore, P. Besmer, Role of dimerization of the membrane-associated growth factor kit ligand in juxtacrine signaling: the S117H mutation affects dimerization and stability-phenotypes in hematopoiesis, J Exp Med 187(9) (1998) 145161.

[15] Y. Jung, J. Wang, A. Havens, Y. Sun, T. Jin, R.S. Taichman, Cell-to-cell contact is critical for the survival of hematopoietic progenitor cells on osteoblasts, Cytokine 32(3-4) (2005) 15562.

[16] F.W. Ruscetti, S. Akel, S.H. Bartelmez, Autocrine transforming growth factor-beta regulation of hematopoiesis: many outcomes that depend on the context, Oncogene 24(37) (2005) 5751-63.

[17] H.P. Gerber, A.K. Malik, G.P. Solar, D. Sherman, X.H. Liang, G. Meng, K. Hong, J.C. Marsters, N. Ferrara, VEGF regulates haematopoietic stem cell survival by an internal autocrine loop mechanism, Nature 417(6892) (2002) 954-8. 
[18] A. Janowska-Wieczorek, M. Majka, J. Ratajczak, M.Z. Ratajczak, Autocrine/paracrine mechanisms in human hematopoiesis, Stem Cells 19(2) (2001) 99-107.

[19] E. Csaszar, W. Wang, T. Usenko, W. Qiao, C. Delaney, I.D. Bernstein, P.W. Zandstra, Blood stem cell fate regulation by Delta-1 mediated rewiring of IL-6 paracrine signaling, Blood (2013).

[20] T. Li, Y. Wu, Paracrine molecules of mesenchymal stem cells for hematopoietic stem cell niche, Bone marrow research 2011 (2011) 353878.

[21] E. Csaszar, D.C. Kirouac, M. Yu, W. Wang, W. Qiao, M.P. Cooke, A.E. Boitano, C. Ito, P.W. Zandstra, Rapid expansion of human hematopoietic stem cells by automated control of inhibitory feedback signaling, Cell Stem Cell 10(2) (2012) 218-29.

[22] M. Majka, A. Janowska-Wieczorek, J. Ratajczak, K. Ehrenman, Z. Pietrzkowski, M.A. Kowalska, A.M. Gewirtz, S.G. Emerson, M.Z. Ratajczak, Numerous growth factors, cytokines, and chemokines are secreted by human CD34(+) cells, myeloblasts, erythroblasts, and megakaryoblasts and regulate normal hematopoiesis in an autocrine/paracrine manner, Blood 97(10) (2001) 3075-85.

[23] D.C. Kirouac, C. Ito, E. Csaszar, A. Roch, M. Yu, E.A. Sykes, G.D. Bader, P.W. Zandstra, Dynamic interaction networks in a hierarchically organized tissue, Mol Syst Biol 6 (2010) 417. [24] R.J. Su, K. Li, X.B. Zhang, P.M. Pan Yuen, C.K. Li, A.E. James, J. Liu, T.F. Fok, Plateletderived growth factor enhances expansion of umbilical cord blood CD34+ cells in contact with hematopoietic stroma, Stem cells and development 14(2) (2005) 223-30.

[25] D.C. Kirouac, G.J. Madlambayan, M. Yu, E.A. Sykes, C. Ito, P.W. Zandstra, Cell-cell interaction networks regulate blood stem and progenitor cell fate, Mol Syst Biol 5 (2009) 293. [26] E. Muller, W. Wang, W. Qiao, M. Bornhauser, P.W. Zandstra, C. Werner, T. Pompe, Distinguishing autocrine and paracrine signals in hematopoietic stem cell culture using a biofunctional microcavity platform, Scientific reports 6 (2016) 31951.

[27] B.P. Mahadik, T.D. Wheeler, L.J. Skertich, P.J. Kenis, B.A. Harley, Microfluidic generation of gradient hydrogels to modulate hematopoietic stem cell culture environment, Advanced healthcare materials 3(3) (2014) 449-58.

[28] D.L. Coutu, J. Cuerquis, R. El Ayoubi, K.A. Forner, R. Roy, M. Francois, M. Griffith, D. Lillicrap, A.M. Yousefi, M.D. Blostein, J. Galipeau, Hierarchical scaffold design for mesenchymal stem cell-based gene therapy of hemophilia B, Biomaterials 32(1) (2011) 295-305. [29] S. Pedron, B.A. Harley, Impact of the biophysical features of a 3D gelatin microenvironment on glioblastoma malignancy, J Biomed Mater Res A (2013).

[30] B.L. Sprague, J.G. McNally, FRAP analysis of binding: proper and fitting, Trends Cell Biol. 15(2) (2005) 84-91.

[31] F.C. Mackintosh, J. Kas, P.A. Janmey, Elasticity of semiflexible biopolymer networks, Phys Rev Lett 75(24) (1995) 4425-4428.

[32] Y.L. Yang, L.M. Leone, L.J. Kaufman, Elastic moduli of collagen gels can be predicted from two-dimensional confocal microscopy, Biophys J 97(7) (2009) 2051-2060.

[33] R.C. Arevalo, J.S. Urbach, D.L. Blair, Size-dependent rheology of type-I collagen networks, Biophys J 99(8) (2010) L65-L67.

42 [34] J. Brandrup, E.H. Immergut, A. Abe, D.R. Bloch, Polymer handbook, Wiley, New York, 43 NY, 1999.

44 [35] M. Rubinstein, R.H. Colby, Polymer physics, Oxford University, New York, 2003.

45 [36] Y. Oono, M. Kohmoto, Renormalization-group theory of transport-properties of polymer-

46 solutions. 1. Dilute-solutions, J Chem Phys 78(1) (1983) 520-528. 
[37] J.R. Lawrence, G.M. Wolfaardt, D.R. Korber, Determination of diffusion-coefficients in biofilms by confocal laser microscopy, Appl Environ Microbiol 60(4) (1994) 1166-1173. [38] I. Leisten, R. Kramann, M.S. Ventura Ferreira, M. Bovi, S. Neuss, P. Ziegler, W. Wagner, R. Knuchel, R.K. Schneider, 3D co-culture of hematopoietic stem and progenitor cells and mesenchymal stem cells in collagen scaffolds as a model of the hematopoietic niche, Biomaterials 33(6) 1736-47.

[39] J.M.T. Ewoldt R.H., Caretta L.M, Experimental challenges of shear rheology with case studies in biological complex fluids, Complex Fluids in Biological Systems, Springer Biological Engineering Series (In Press (2014)).

[40] F. Chambon, H.H. Winter, Linear Viscoelasticity at the Gel Point of a Cross-Linking Pdms with Imbalanced Stoichiometry, J Rheol 31(8) (1987) 683-697.

[41] A. Erikson, H.N. Andersen, S.N. Naess, P. Sikorski, C.D. Davies, Physical and chemical modifications of collagen gels: Impact on diffusion, Biopolymers 89(2) (2008) 135-143. [42] R. Ishino, K. Minami, S. Tanaka, M. Nagai, K. Matsui, N. Hasegawa, R.G. Roeder, S. Asano, M. Ito, FGF7 supports hematopoietic stem and progenitor cells and niche-dependent myeloblastoma cells via autocrine action on bone marrow stromal cells in vitro, Biochem Biophys Res Commun 440(1) (2013) 125-31. [43] C.B. Hutson, J.W. Nichol, H. Aubin, H. Bae, S. Yamanlar, S. Al-Haque, S.T. Koshy, A. Khademhosseini, Synthesis and characterization of tunable poly(ethylene glycol): gelatin methacrylate composite hydrogels, Tissue Eng Part A 17(13-14) (2011) 1713-23. [44] J.W. Shin, A. Buxboim, K.R. Spinler, J. Swift, D.A. Christian, C.A. Hunter, C. Leon, C. Gachet, P.C. Dingal, I.L. Ivanovska, F. Rehfeldt, J.A. Chasis, D.E. Discher, Contractile forces sustain and polarize hematopoiesis from stem and progenitor cells, Cell Stem Cell 14(1) (2014) 81-93.

[45] J.S. Choi, B.A.C. Harley, Marrow-inspired matrix cues rapidly affect early fate decisions of hematopoietic stem and progenitor cells, Science Advances 3(1) (2017) e1600455. [46] E. Altrock, C.A. Muth, G. Klein, J.P. Spatz, C. Lee-Thedieck, The significance of integrin ligand nanopatterning on lipid raft clustering in hematopoietic stem cells, Biomaterials 33(11) (2012) 3107-18.

[47] S. Mishima, A. Nagai, S. Abdullah, C. Matsuda, T. Taketani, S. Kumakura, H. Shibata, H. Ishikura, S.U. Kim, J. Masuda, Effective ex vivo expansion of hematopoietic stem cells using osteoblast-differentiated mesenchymal stem cells is CXCL12 dependent, European journal of haematology 84(6) (2010) 538-46. [48] Y. Nakamura, F. Arai, H. Iwasaki, K. Hosokawa, I. Kobayashi, Y. Gomei, Y. Matsumoto, H. Yoshihara, T. Suda, Isolation and characterization of endosteal niche cell populations that regulate hematopoietic stem cells, Blood 116(9) (2010) 1422-32.

[49] S.J. Morrison, D.T. Scadden, The bone marrow niche for haematopoietic stem cells, Nature 505(7483) (2014) 327-34.

[50] P.E. Boulais, P.S. Frenette, Making sense of hematopoietic stem cell niches, Blood 125(17) (2015) 2621-9.

41 [51] S. Pedron, E. Becka, B.A. Harley, Regulation of glioma cell phenotype in 3D matrices by 42 hyaluronic acid, Biomaterials 34(30) (2013) 7408-17.

43 [52] I. Kim, S. He, O.H. Yilmaz, M.J. Kiel, S.J. Morrison, Enhanced purification of fetal liver 44 hematopoietic stem cells using SLAM family receptors, Blood 108(2) (2006) 737-744. 
1 [53] B.M. Sagar, S. Rentala, P.N. Gopal, S. Sharma, A. Mukhopadhyay, Fibronectin and laminin 2 enhance engraftibility of cultured hematopoietic stem cells, Biochem Biophys Res Commun 3 350(4) (2006) 1000-5. 